\title{
Breaking the scale invariance of the primordial power spectrum in Hořava-Lifshitz cosmology
}

\author{
Kazuhiro Yamamoto ${ }^{1}$, Tsutomu Kobayashi², Gen Nakamura ${ }^{1}$ \\ ${ }^{1}$ Department of Physical Science, Hiroshima University, Higashi-Hiroshima 739-8526, Japan \\ ${ }^{2}$ Department of Physics, Waseda University, Okubo 3-4-1, Shinjuku, Tokyo, 169-8555, Japan
}

\begin{abstract}
We study the spectral tilt of primordial perturbations in Hořava-Lifshitz cosmology. The uniform approximation, which is a generalization of the familiar Wentzel-Kramers-Brillouin (WKB) method, is employed to compute the spectral index both numerically and analytically in a closed form. We clarify how the spectral index depends on the inflation model and parameters in the modified dispersion relation.

PACS numbers: $98.80 . \mathrm{Cq}$
\end{abstract}

\section{INTRODUCTION}

A power-counting renormalizable theory of gravity, proposed recently by Hořava [1], has attracted much attention and generated a trend in quantum gravity. The essential aspect of the theory is broken Lorentz invariance in the ultraviolet (UV), where it exhibits a Lifshitzlike anisotropic scaling, $t \rightarrow \ell^{z} t, \vec{x} \rightarrow \ell \vec{x}$, with the dynamical critical exponent $z=3$. Modification to general relativity thus introduced in the high energy regime brings interesting consequences in cosmology [2], including the generation of chiral gravitational waves from inflation [3], a bouncing scenario [4], a possible candidate for dark matter [5], and others [6]. Since signals from the early universe could be observed for instance through the cosmic microwave background (CMB) temperature anisotropies and the stochastic gravitational wave background, it is important to study the dynamics cosmological perturbations in Hořava-Lifshitz (HL) cosmology (see, e.g., Ref. 7] and references therein). However, it should be noted that problems of HL gravity are still under debate [ㅁ, $\underline{6}, \underline{9}]$.

One of the basic ingredients in today's cosmology is the almost scale-invariant power spectrum of primordial perturbations generated quantum mechanically in the early universe, and what is equally important is a small deviation from the exact scale-invariance. Inflation involves a natural mechanism to generate almost scale-invariant cosmological perturbations, and indeed this fact together with observations of the CMB and the large scale structure (LSS) in the universe provides increasing support for inflation. The scale-invariance in this case essentially relies upon the almost constant Hubble rate, and a slight deviation from the exact de Sitter expansion is responsible for the small tilt of the spectrum.

Another interesting mechanism to generate scaleinvariant cosmological perturbations was recently pointed out by Mukohyama 10 in connection with HL cosmology. (See also Refs. 11, 12, 13, 14, 15, 16] for cosmological perturbations in HL gravity.) The anisotropic scaling in the UV allows us to include higher spatial derivative terms in a scalar field Lagrangian, modifying the dispersion relation into the form $\omega^{2} \propto k^{6}$ in the early universe, which results in scale-invariant scalar field perturbations. In contrast to the standard mechanism, the spectrum in Mukohyama's scenario does not depend on the Hubble rate explicitly but is determined only through some model parameter. Therefore, the new mechanism liberates inflation models from those with a very flat potential. In Mukohyama's scenario, the dispersion relation which is not exactly of the form $\omega^{2} \propto k^{6}$ will break the scale-invariance.

In this paper we compute a slightly tilted spectrum of quantum fluctuations generated in HL cosmology, evaluating accurately the spectral index by means of the uniform approximation [17, 18, 19]. The uniform approximation is a generalized Wentzel-Kramers-Brillouin (WKB) method, which is mathematically controlled and systematically extendable. To the best of the authors' knowledge, the uniform approximation has not been used so far for models with modified dispersion relations. We consider a power-law/slow-roll inflationary background in HL cosmology, which provides a transparent example that allows for analytical and numerical calculations. Although our primary motivation comes from HL cosmology, we believe that the approach presented in this paper has a wide range of applications in different scenarios of cosmology.

\section{UNIFORM APPROXIMATION}

We consider a quantum field whose Fourier coefficients obey the equation of motion,

$$
\varphi_{k}^{\prime \prime}(\eta)+\left[k_{\text {eff }}^{2}(\eta)-\frac{a^{\prime \prime}}{a}\right] \varphi_{k}(\eta)=0,
$$

where the prime denotes the differentiation with respect to conformal time $\eta, a(\eta)$ is the scale factor, and $k_{\text {eff }}^{2}(\eta)$ is the effective frequency [3, 10]. The field $\varphi_{k}$ will be a gravitational wave (multiplied by the scale factor) [3] and a scalar field perturbation (multiplied by a scale factor) [10]. Following the prescription of the uniform approximation [17, 18, 19], we rewrite the above equation as

$$
\varphi_{k}^{\prime \prime}(\eta)=[g(\eta)+q(\eta)] \varphi_{k}(\eta)
$$


where we defined

$$
g(\eta)=-k_{\mathrm{eff}}^{2}(\eta)+\frac{a^{\prime \prime}}{a}+\frac{1}{4 \eta^{2}},
$$

and $q(\eta)=-1 / 4 \eta^{2}$, which guarantees the convergence of the approximation. This method provides a single approximate solution $\varphi_{k}(\eta)=[g(\eta) / \zeta(\eta)]^{-1 / 4}\left[\alpha_{k} \operatorname{Ai}(\zeta)+\beta_{k} \operatorname{Bi}(\zeta)\right]$, with $\zeta(\eta):= \pm\left[ \pm(3 / 2) \int_{\bar{\eta}}^{\eta} \sqrt{ \pm g\left(\eta_{1}\right)} d \eta_{1}\right]^{2 / 3}$, where + for $\eta>\bar{\eta}$, - for $\eta<\bar{\eta}$, respectively, and $\bar{\eta}$, which corresponds to the effective horizon-crossing time, is defined by $g(\bar{\eta})=0$, and $\operatorname{Ai}(z)$ and $\operatorname{Bi}(z)$ are the Airy functions. Choosing $\alpha_{k}=\sqrt{\pi / 2}$ and $\beta_{k}=-i \sqrt{\pi / 2}$, the mode function $\varphi_{k}$ is made to satisfy the Wronskian normalization condition $\varphi_{k} \varphi_{k}^{* \prime}-\varphi_{k}^{\prime} \varphi_{k}^{*}=i$ and has the asymptotic early-time behavior $\varphi_{k}(\eta) \simeq\left[2 k_{\text {eff }}(\eta)\right]^{-1 / 2} \times \exp \left[-i \int_{\bar{\eta}}^{\eta} k_{\text {eff }}\left(\eta_{1}\right) d \eta_{1}\right]$, for $\eta \ll \bar{\eta}$.

Using the asymptotic formula of the Airy function $\operatorname{Ai}(z) \simeq(2 \sqrt{\pi})^{-1} z^{-1 / 4} e^{-2 z^{3 / 2} / 3}$ and $\operatorname{Bi}(z) \simeq$ $(\sqrt{\pi})^{-1} z^{-1 / 4} e^{2 z^{3 / 2} / 3}$ for $|z| \rightarrow \infty$ with $-\pi<\arg z<\pi$, we find the asymptotic formula of the mode function after the horizon crossing:

$$
\varphi_{k}(\eta) \simeq-\frac{i}{\sqrt{2}}[g(\eta)]^{-1 / 4} \exp \left[\int_{\bar{\eta}}^{\eta} \sqrt{g\left(\eta_{1}\right)} d \eta_{1}\right] .
$$

Thus, we finally obtain the power spectrum $P_{\varphi}(k, \eta)=$ $\left|\varphi_{k}(\eta) / a(\eta)\right|^{2}$, and the spectral index of the quantum fluctuations can be estimated from $d \ln P_{\varphi}(k, \eta) / d \ln k$, which leads to

$$
n_{\mathrm{ini}} \simeq \lim _{\eta \rightarrow \eta_{\max }} 2 k \frac{d}{d k} \int_{\bar{\eta}(k)}^{\eta} \sqrt{g\left(\eta_{1}\right)} d \eta_{1} .
$$

This provides a closed formula for the spectral index. Note, however, that this formula cannot be used for the cases in which the fluctuations reenter the horizon and the horizon crossing takes place again.

\section{POWER-LAW INFLATION}

For simplicity, let us consider power-law inflation [20], for which $a(t) \propto t^{1+n}$ in terms of proper time $t$ and $a(\eta)=1 /\left(-H_{*} \eta\right)^{1+1 / n}$ in terms of conformal time $\eta(-\infty<\eta<0)$, with $n>0$. We first study a simple case,

$$
k_{\mathrm{eff}}^{2}(\eta)=k^{2}\left(\frac{k^{2}}{M^{2} a^{2}}\right)^{\mu} .
$$

In this case, it is easy to find the superhorizon behavior in the uniform approximation:

$$
\begin{aligned}
& \varphi_{k}(\eta) \approx-i a(\eta) \mathcal{A} e^{-\nu}(3+2 / n)^{\nu-1 / 2} k^{-(1+\mu) \nu}, \\
& \mathcal{A}:=H_{*}^{\nu-1 / 2} M^{\mu \nu}, \quad \nu:=\frac{1+3 n / 2}{\mu+n(\mu+1)},
\end{aligned}
$$

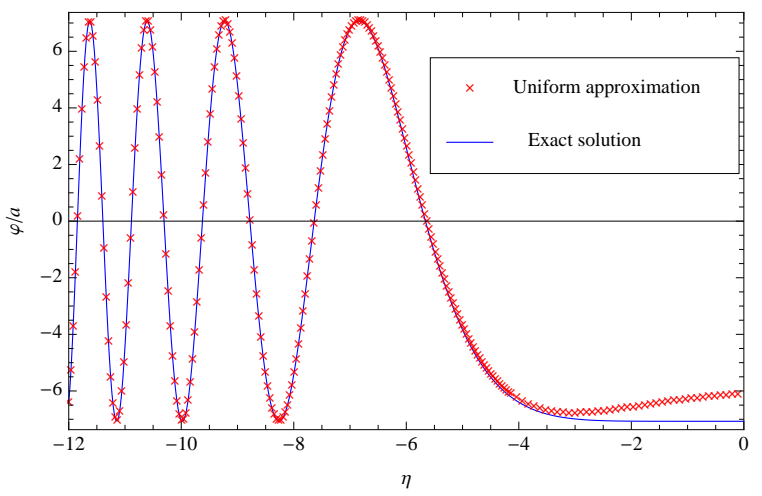

FIG. 1: Growing mode solution to Eq. (1), obtained by employing the uniform approximation, is compared to the exact result. The parameters are given by $\mu=2, n=3, H_{*}=1$, and $k^{3} / M^{2}=10^{-2}$. The horizon-crossing time is $\bar{\eta}=-4.14$.

where $\mathcal{A}$ defines the typical amplitude of the fluctuation. In the de Sitter limit $n \rightarrow \infty$ with the usual dispersion relation $\mu=0$, we have $\mathcal{A}=H_{*}$. For $\mu=2$ we find $\mathcal{A}=M$, and thus the result of [10] is reproduced. Fortunately, for the dispersion relation (6), Eq. (1) can be solved exactly, and so we can compare the exact result with the approximate one. The appropriately normalized solution to Eq. (1) is given by

$$
\varphi_{\text {exact }}(\eta)=\frac{1}{2} \sqrt{\frac{n \pi}{\mu+n(\mu+1)}}(-\eta)^{1 / 2} H_{\nu}^{(1)}(y(\eta)),
$$

where $H_{\nu}^{(1)}$ is the Hankel function of the first kind and $y(\eta):=\int_{\eta}^{0} k_{\mathrm{eff}}\left(\eta_{1}\right) d \eta_{1}$. This then implies that $\left|\varphi_{k}\right|=$ $\mathcal{C}\left|\varphi_{\text {exact }}\right|$ at $\eta \approx 0$, where $\mathcal{C}:=\sqrt{2 \pi} e^{-\nu} \nu^{\nu-1 / 2} / \Gamma(\nu)$. In the de Sitter limit, $n \rightarrow \infty$, for $\mu=0$ we find $\mathcal{C}=$ $3 \sqrt{2} e^{-3 / 2} \simeq 0.95$, while for $\mu=2$ we have $\mathcal{C}=\sqrt{2 / e} \simeq$ 0.86 irrespective of $n$. It is important to note that $\mathcal{C}$ gives rise to a $k$-independent correction to the amplitude, and therefore this does not affect the computation of the spectral index. The exact result and the approximate one are compared in Fig. 1 in the case of $\mu=2$.

Now we are in position to study the spectral index for the above model. Eq. (5) or (7) gives

$$
n_{\text {ini }}+3=\frac{\mu-2}{\mu+n(\mu+1)},
$$

which leads to the scale-invariant spectrum $n_{\text {ini }}=-3$ when $\mu=2$, as expected [10]. The result (9) is consistent with what was obtained in a different way by matching two asymptotic solutions at horizon crossing [15]. Since the slow-roll parameter $\epsilon\left(:=-H^{\prime} / a H^{2}\right)$ is explicitly given by $\epsilon=1 /(1+n)$ for power-law inflation, Eq. (9) can be written as $n_{\text {ini }}+3 \simeq-\epsilon(2-\mu) /(1+\mu)$ to leading order in the slow-roll parameter. One can confirm that this reproduces the standard result for $\mu=0$. Note also that $n_{\text {ini }}$ approaches -3 in the limit $n \rightarrow \infty(\epsilon \rightarrow 0)$ irrespective of the value $\mu$. This means that in order to break 
the scale invariance of the primordial spectrum we need the background universe which is not exactly de Sitter and $\mu \neq 2$.

Next, let us study the case of HL cosmology, for which

$$
k_{\text {eff }}^{2}(\eta)=k^{2}\left(\frac{k^{4}}{M^{4} a^{4}}+\alpha_{1} \frac{k^{3}}{M^{3} a^{3}}+\alpha_{2} \frac{k^{2}}{M^{2} a^{2}}+1\right) .
$$

In the case of gravitational waves, $\alpha_{1}$ is non-zero [3] , while $\alpha_{1}=0$ for a scalar field [10]. For this dispersion relation Eq. (5) reduces to

$$
\begin{aligned}
n_{\mathrm{ini}} & =\frac{\kappa n}{1+n} \times \\
& \int_{0}^{\bar{z}} \frac{-z^{-(2+n) /(1+n)}\left[z^{2} f(z)\right]^{\prime} d z}{\sqrt{\left(n^{-1}+3 / 2\right)^{2} \kappa^{-2} z^{-2 n /(1+n)}-f(z)}}
\end{aligned}
$$

where $f(z):=z^{4}+\alpha_{1} z^{3}+\alpha_{2} z^{2}+1, \quad \kappa \quad:=$ $\left(M / H_{*}\right)(k / M)^{1 /(1+n)}$ and we defined a new variable $z$ by $-H_{*} \eta=(M z / k)^{n /(1+n)}$. The prime in Eq. (11) stands for the differentiation with respect to $z$. The upper limit of integration $\bar{z}$ satisfies

$$
(1 / n+3 / 2)^{2} \kappa^{-2} \bar{z}^{-2 n /(1+n)}-f(\bar{z})=0 .
$$

Figure 2 shows the contour of $n_{\text {ini }}$ in the $\kappa$ and $1 / n$ plane, obtained from a numerical integration of Eq. (11). The parameters are given by $\alpha_{1}=0$ and $\alpha_{2}=1$ for the left panel, which corresponds to a scalar field perturbation of [10], $\alpha_{1}=2, \alpha_{2}=1$ and $\alpha_{1}=-2, \alpha_{2}=1$, respectively, for the center and right panels. The latter two cases correspond to chiral gravitational waves analyzed in [3].

The result is understood in an analytic way as follows.

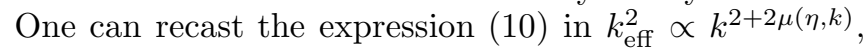
where $\mu(\eta, k)=(1 / 2) d \ln f / d \ln z$, and so $\mu$ is dependent on $\eta$ and $k$ only through $z$. For the case $\kappa \ll 1$, the solution to Eq. (12) is such that $\bar{z} \gg 1$, and thus we have $\bar{z} \simeq\left[\kappa^{-1}\left(n^{-1}+3 / 2\right)\right]^{(1+n) /(2+3 n)}$ and $\mu(k, \bar{\eta}) \simeq 2-$ $\alpha_{1} /(2 \bar{z})+\left(\alpha_{1}^{2}-2 \alpha_{2}\right) /\left(2 \bar{z}^{2}\right)$. Then, Eq. (9) gives

$$
n_{\text {ini }}+3 \simeq-\left(\frac{\alpha_{1}}{2 \bar{z}}-\frac{\alpha_{1}^{2}-2 \alpha_{2}}{2 \bar{z}^{2}}\right) \frac{1}{2+3 n} .
$$

This result applies to the case where the horizon crossing occurs in the UV regime, $k_{\text {eff }}^{2} \sim k^{6}$. While, for the case $\kappa \gg 1$, the solution to Eq. (12) is given by $\bar{z} \ll 1$, which corresponds to the case where the horizon crossing takes place in the infrared (IR) regime, $k_{\text {eff }}^{2} \sim k^{2}$. In this case, we may write $\bar{z} \simeq\left[\kappa^{-1}\left(n^{-1}+3 / 2\right)\right]^{(1+n) / n}$ and $\mu(k, \bar{\eta}) \simeq$ $\alpha_{2} \bar{z}^{2}+(3 / 2) \alpha_{1} \bar{z}^{3}$. Then, Eq. (9) yields

$$
n_{\mathrm{ini}} \simeq-3\left(1-\frac{\alpha_{2} \bar{z}^{2}+3 \alpha_{1} \bar{z}^{3} / 2}{n}\right)\left(1+\frac{2}{3 n}\right) .
$$

The approximate formula reproduces the qualitative behavior of the numerical result. The above results suggest that we must require small $\kappa(\lesssim 1)$ or large $n(\gtrsim 10)$, in order to have a spectrum that is almost scale-invariant but slightly deviated from the scale-invariant one. Note that

$$
\begin{gathered}
\kappa=\frac{10^{6}}{\left(1.5 \times 10^{57}\right)^{1 /(1+n)}}\left(\frac{M}{10^{19} \mathrm{GeV}}\right)^{n /(1+n)} \times \\
\left(\frac{10^{15} \mathrm{GeV}}{H_{*}}\right)\left(\frac{k}{\mathrm{Mpc}^{-1}}\right)^{1 /(1+n)} .
\end{gathered}
$$

The sign of $\alpha_{1}$ signals different chiral modes of primordial gravitational waves [3], and hence the spectral tilt of the gravitational waves is different for right-handed and left-handed modes. The above result shows explicitly the dependence of the spectral index on model parameters. Our result also gives a hint on the scenario of [10], in which quantum fluctuations of a scalar field can be the origin of curvature perturbations by promoting the former to the latter, e.g., via the curvaton mechanism [21].

\section{SLOW-ROLL INFLATION}

Let us move on to more general slow-roll inflation models. We continue to study Eq. (1), but now with $a^{\prime \prime} / a$ replaced by $a^{\prime \prime} / a \rightarrow C(\eta)^{2} / \eta^{2}=2 a^{2} H^{2}(1+E)$. Here, $E$ is some general function whose explicit form depends on the problem under consideration: $E=-\epsilon / 2$ for a tensor perturbations and a scalar field perturbation in the slow-roll regime; $E \simeq \epsilon-3 \delta / 2$, where $\delta(:=-\ddot{\phi} / H \dot{\phi})$ is another slow-roll parameter, for a scalar perturbation in conventional slow-roll inflation models, where $\phi$ is the inflaton field and the dot denotes the differentiation with respect to the proper time. However, a further careful investigation is necessary for a scalar (curvature) perturbation in HL cosmology. For example, the $k^{6}$ term in the dispersion relation disappears under the assumption of the detailed balance condition, according to the prescription of Ref. [14]. References [7, 16] investigate a different case without the detailed balance condition but with the projectability condition.

Assuming the dispersion relation (6), a similar calculation for the right hand side of Eq. (5) yields $n_{\mathrm{ini}}+3 \simeq$ $-(3 \epsilon) /(1+\mu)+\epsilon / 3-4 E / 3$ to lowest order in the slowroll parameters, where we assumed that $E$ is of order of the slow-roll parameters and used the relation $\eta \simeq 1 /[-H a(1-\epsilon)]$. In the case of $\mu=0$, the well-known result is reproduced for the tensor and scalar perturbations. Similarly to the above, we can also derive formulas for the dispersion relation (10). Hereafter, we define $\bar{z}$ by the root of $f(\bar{z})=\left(\bar{H}^{2} / \bar{z}^{2} M^{2}\right)(9 / 4+2 E-\epsilon / 2)$, where $\bar{H}$ is the value of $H$ at the horizon-crossing time. For the case $M / \bar{H} \ll 1$, we find $\bar{z} \simeq(3 \bar{H} / 2 M)^{1 / 3} \gg 1$ and

$$
n_{\mathrm{ini}}+3 \simeq-\left(1+\frac{\alpha_{1}}{6 \bar{z}}+\frac{2 \alpha_{2}-\alpha_{1}^{2}}{6 \bar{z}^{2}}\right) \epsilon+\frac{1}{3} \epsilon-\frac{4}{3} E,
$$

while for the case $M / \bar{H} \gg 1$ we have $\bar{z} \simeq(3 \bar{H} / 2 M) \ll 1$ and

$$
n_{\mathrm{ini}}+3 \simeq-3\left(1-\alpha_{2} \bar{z}^{2}-\frac{3}{2} \alpha_{1} \bar{z}^{3}\right) \epsilon+\frac{1}{3} \epsilon-\frac{4}{3} E .
$$



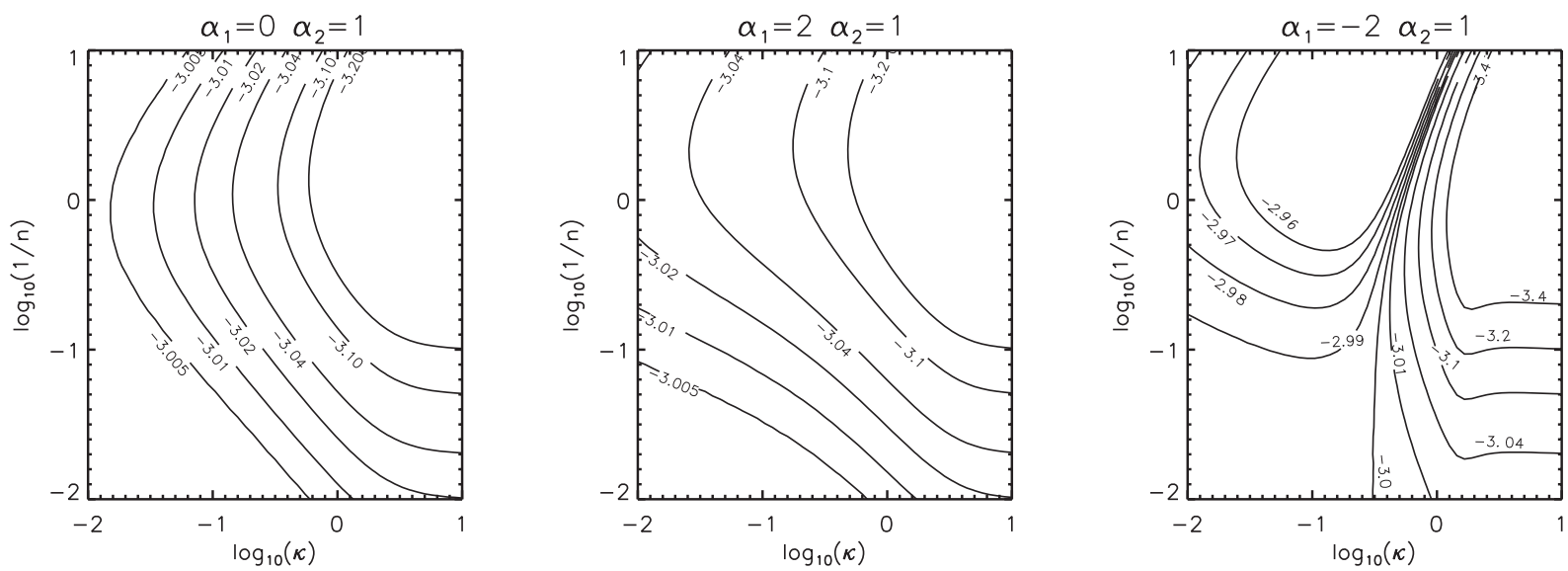

FIG. 2: (a: Left) Contours of $n_{\text {ini }}$ on the $1 / n$ and $\kappa$ plane. The parameters are $\alpha_{1}=0$ and $\alpha_{2}=1$. The level of the contour is shown in the panel. (b: Center) Same as (a), but with $\alpha_{1}=2$ and $\alpha_{2}=1$. (c: Right) Same as (a), but with $\alpha_{1}=-2$ and $\alpha_{2}=1$.

One can check that these results are consistent with those of the power-law inflationary background with large value of $n+1(=1 / \epsilon)$, by taking the expression for the gravitational waves and the scalar field, i.e., $E=-\epsilon / 2$.

In [3], it was pointed out that the evolution of primordial gravitational waves is different depending on the parity mode. From Eqs. (16) and (17), the difference of the spectral index is given by

$$
\Delta n_{\mathrm{ini}}=\left\{\begin{array}{ll}
-\frac{\epsilon\left|\alpha_{1}\right|}{3}\left(\frac{2 M}{3 \bar{H}}\right)^{1 / 3} & (M / \bar{H} \ll 1) \\
9 \epsilon\left|\alpha_{1}\right|\left(\frac{3 \bar{H}}{2 M}\right)^{3} & (M / \bar{H} \gg 1)
\end{array} .\right.
$$

The difference might not be negligible for $M / \bar{H} \ll 1$, which corresponds to the case where the horizon crossing takes place in the UV regime $\left(k_{\text {eff }}^{2} \sim k^{6}\right)$. However, the difference is small for $M / \bar{H} \gg 1$, which is the case where the horizon-crossing occurs in the IR regime $\left(k_{\text {eff }}^{2} \sim k^{2}\right)$. If we assume a scenario of Hořava-Lifshitz cosmology, one can predict the signal of gravitational waves, to be compared with observations (e.g., Ref. [22]).

Now we consider curvature perturbations in the scenario of [10] by setting $\alpha_{1}=0$ and $E=-\epsilon / 2$. Provided that the spectral tilt of scalar field perturbations is directly translated into that of curvature perturbations, we have

$$
n_{\mathrm{ini}}+3 \simeq\left\{\begin{array}{ll}
-\frac{\epsilon \alpha_{2}}{3}\left(\frac{2 M}{3 \bar{H}}\right)^{2 / 3} & (M / \bar{H} \ll 1) \\
-2 \epsilon+\frac{27 \epsilon \alpha_{2}}{4} \frac{\bar{H}^{2}}{M^{2}} & (M / \bar{H} \gg 1)
\end{array} .\right.
$$

The CMB measurement by the WMAP satellite and LSS of galaxies provide a stringent constraint on the primordial spectral index. For example, the WMAP team reported $n_{\text {ini }}+3 \sim-0.04 \pm 0.013$ based on the $\Lambda \mathrm{CDM}$ model 23], combined with Ia supernovae observations. This gives a constraint on the inflation model and the parameters in the scalar field Lagrangian, assuming that the almost scale-invariant spectrum we observe is originated from the Hořava-Lifshitz scalar field [10].

\section{CONCLUSIONS}

In this paper, we have studied the spectral tilt of quantum fluctuations in HL cosmology, employing the uniform approximation. In the power-law inflationary background we have obtained the spectral index numerically and analytically. The deviation from the scale invariant spectrum is described by the parameters $\alpha_{1}$ and $\alpha_{2}$ of the model, as well as the background evolution, i.e., $n$. The case of a general slow-roll inflationary background was also investigated. In order for Mukohyama's scenario [10] to be successful, the spectral index, given in terms of the model parameters by Eq. (19), is required to be $\sim-0.04$.

\section{Acknowledgments}

This work was supported by a Grant-in-Aid for Scientific research of the Japanese Ministry of Education, Culture, Sports, Science and Technology (No. 21540270), and in part by the Japan Society for Promotion of Science (JSPS) Core-to-Core Program "International Research Network for Dark Energy." We would like to thank S. Mukohyama, G. Niz, T. Padilla, and E. Saridakis for useful communications. TK is supported by the JSPS under Contract No. 01642. He is grateful for the kind hospitality of the theoretical astrophysics group at Hiroshima University, where this work was initiated. 
[1] P. Hořava, Phys. Rev. D 79, 084008 (2009).

[2] G. Calcagni, arXiv:0904.0829 E. Kiritsis and G. Kofinas, arXiv:0904.1334.

[3] T. Takahashi and J. Soda, Phys. Rev. Lett. 102, 231301 (2009)

[4] R. Brandenberger, arXiv:0904.2835.

[5] S. Mukohyama, arXiv:0905.3563 S. Mukohyama, arXiv:0906.5069.

[6] S. Mukohyama et al. arXiv:0905.0055, E. N. Saridakis, arXiv:0905.3532, M-I Park, arXiv:0906.4275 M. Minamitsuji, arXiv:0905.3892; S. Nojiri and S. D. Odintsov, arXiv:0905.4213

[7] A. Wang and R. Maartens, arXiv:0907.1748

[8] C. Charmousis, G. Niz, A. Padilla, P. M. Saffin, arXiv:0905.2579 D. Blas, O. Pujolas, S. Sibiryakov, arXiv:0906.3046

[9] C. Bogdanos and E. N. Saridakis, arXiv:0907.1636

[10] S. Mukohyama, JCAP 0906001 (2009), arXiv:0904.2190.

[11] Y. S. Piao, arXiv:0904.4117
[12] X. Gao, arXiv:0904.4187.

[13] B. Chen, S. Pi, J. Z. Tang, arXiv:0905.2300

[14] X. Gao et al., arXiv:0905.3821

[15] Yi-Fu Cai, X. Zhang, arXiv:0906.3341.

[16] T. Kobayashi, Y. Urakawa and M. Yamaguchi, arXiv:0908.1005

[17] J. Martin, D. J. Schwartz, Phys. Rev. D 67, 083512 (2003).

[18] S. Habib et al.,Phys. Rev. Lett. 89, 281301 (2002).

[19] S. Habib et al., Phys. Rev. D 70, 083507 (2004).

[20] F. Lucchin, S. Matarrese, Phys. Rev. D 32, 1316 (1985).

[21] K. Enqvist and M. S. Sloth, Nucl. Phys. B 626, 395 (2002); D. H. Lyth and D. Wands, Phys. Lett. B 524, 5 (2002); T. Moroi and T. Takahashi, Phys. Lett. B 522, 215 (2001) [Erratum-ibid. B 539, 303 (2002)].

[22] S. Koh, arXiv:0907.0850.

[23] E. Komatsu et al., Astrophys. J. Suppl. 180, 330 (2009). 